\title{
Linear Position Sensors - A Brief Guide of Use of the Most Common Types
}

\author{
M. Papoutsidakis \\ Dept. of Automation Eng. \\ Piraeus University \\ Of Applied Sciences, \\ Athens, Greece
}

\author{
A. Chatzopoulos \\ Dept. of Automation Eng. \\ Piraeus University \\ Of Applied Sciences, \\ Athens, Greece
}

\author{
C. Drosos \\ Dept. of Computer Science \\ University of Thessaly, \\ Lamia, Greece
}

\begin{abstract}
In this paper, we gave information about introduction to the linear position sensors, their history and classification. In the content of this document, main parameter for this type of sensors are discussed while considering their application areas. Primary advantages or disadvantages of this kind of sensors are determined by their operating style and principle. Simulation result carried out in real life represents the comparison among these sensors. Those simulations are supplied with corresponding theoretical equations and formulas. Additionally, a novel read-out signal conditioning circuitry for Linear Variable Differential Transformers (LVDT) and Magnetostriction phenomenon are discussed and provided with specific instances.
\end{abstract}

\section{Keywords}

Linear position sensors, LVDT, magnetostrictive sensors, linear encoders, signal conditioning

\section{INTRODUCTION}

Sensors that measure linear position are found in many industrial applications [1], such as computer numerical control for machine tools, liquid-level monitoring, machine pressing, precise hydraulic systems, and automobile assembling. Several technological possibilities, all varying in measurement range, precision, response speed, and cost [2], have been developed for this purpose. A summary of the characteristics of current commercial linear position sensors is presented in Table I. The potentiometer is an inexpensive linear position sensor, which due to the noise caused by the contact between the wiper and the resistive element, has limited measurement precision. Linear variable differential transformers (LVDTs) exploit the change in the magnetic field coupling between a set of transformers caused by the displacement of a movable ferromagnetic core, providing a contactless and absolute measurement. Although accurate over a small measuring range, their linearity rapidly decreases with ranges above approximately $100 \mathrm{~mm}$. The laser interferometer is the state-of-the-art position sensor, reaching, under controlled light and vibration conditions, an accuracy of $0.1 \mathrm{ppm}$ and a resolution in the nanometer range; for this reason, it is often used to calibrate less-precise sensors.

There are different facts about the history of the sensors depending upon the classification. For instance, the widely spread type of the sensors in industry is electric thermostat. Its invention history consists of multiple stages. Firstly, this sensor was invented by Cornelis Drebbel in the beginning of the 17th century in England in order to create simple feedback control system. Turning to the other type of sensor, namely motion sensor it was firstly designed for the alarm systems by Samuel Bango in 1950s. Its working principle relied upon the analysis of the ultrasonic frequencies via Doppler effect.
Sensors can be come across in either nature or industry. Most popular and interesting application of sensor in nature is the existence of five crucial senses in human. This includes sight, taste, smell, touch, and hearing. Turning to the industry, sensors play an inevitably important role in process. For instance, measurement of the parameters is required in each stage in order to decide which action should be applied.

\section{TABLE I}

PERFORMANCE OF LINEAR POSITION SENSORS: THE ACCURACY FIGURE

\section{CORRESPONDS TO THE MAXIMUM ERROR IN A 1000- MM RANGE}

FOR TYPICAL COMMERCIAL SENSORS. (*) FOR THE LVDT,

THE RANGE CORRESPONDS TO 100 MM

\begin{tabular}{|l|llr|}
\hline Technology & Abs/Inc & Range & Accuracy $(\mu \mathrm{m})$ \\
\hline Potentiometric & Abs & Medium & 400 \\
LVDT & Abs & Small & $250^{\circ}$ \\
Magnetostrictive & Abs & Large & 200 \\
Optical encoder & Inc & Large & 5 \\
Laser interferometer & Inc & Very large & 0.1 \\
\hline
\end{tabular}

The most common precision linear position sensor is the optical encoder, whose operation is based on counting marks arranged on a grating or scale. It has excellent linearity (ultimately related to the precision in the fabrication of the pitch in the grating), with a typical accuracy of $5 \mu \mathrm{m}$ or better, and a resolution of $1 \mu \mathrm{m}$, over a very wide measurement range (up to several meters). Linear position encoders are usually based on the reflection or diffraction of infrared signals on the marks of the grating. However, other non-optical possibilities exist; for example, capacitive encoders have been described. The main disadvantage of encoders is that they are costly and that their incremental nature is problematic in cases of power loss or measurement corruption; in this situation, the sensor must be moved to a reference mark for position retrieval. Particularly, in machine tool operation, this may mean damage to the piece that was being manufactured. Being an optical method of measurement, the encoder must be sealed to protect it from typical machine tool contaminants such as shavings and metalworking coolant fluid.

Magnetostrictive (MS) position sensors, which started as a byproduct of the MS delay lines [4] are used in the 1960s as computer memories, offer an interesting alternative to optical encoders. An MS sensor finds the linear position of a mobile element by computing the time delay of an ultrasonic wave generated at the position of the cursor and transmitted by a waveguide to a receiver placed at one end of this element [2]. 
The ultrasonic signal is created in the waveguide without contact by the MS effect. Unlike optical encoders, MS sensors provide absolute position measurement. However, their relatively high nonlinearities (typically $200 \mu \mathrm{m}$ over a 1000 $\mathrm{mm}$ range) limit their usage to applications with lessdemanding precision such as liquid-level sensing.

The goal of this paper is to explore the physical features that limit the performance of linear position sensors and to comprehence how their accuracy can be increased.

The remainder of this paper is organized as follows: The principle of operation and the relevant theory for highprecision measurement of position with linear variable displacement transformer are described in Section III. The implementation details of the magnetostrictive are discussed in Section IV, whereas the empirical data of its performance are presented in Section VI. Final comments and conclusions are stated in Section VII and VIII.

\section{LINEAR VARIABLE DISPLACEMENT TRANSFORMER}

A linear variable differential transformer, also known as LVDT, is the sensor detecting the displacement, namely position, which is linear. Inductive transducer (LVDT), is one of the useful transducer for measurement and instrumentation systems. The behavior of the LVDT exhibits in terms of high linearity and robustness. Data can be got from these sensors in type of either voltage/current signal or frequency. LVDTs are also classified according to the output type into three subdivisions: alternating current (AC/AC), direct current(DC/DC), and frequency. This kind of sensors was originally patented by George B. Hoadly in 1930s. However, there are not any considerably changes between the previous model and the current one.

Turning to the construction, the main body of this device is cylindrical bobbin. Choosing this component requires some parameters that should be considered. For instance, it should be made of non-magnetic and insulating material. Primary winding is placed in the center of above mentioned bobbin in which manner that its tips are fed with the alternating current source. In addition, secondary windings, which have identical number of turns, are serially located in both sides of the primary winding. The ends of these windings are connected to the measurement device to get the output data. As a measurement device, voltmeter is mostly preferred. To supply the motion inside the bobbin, the movable soft iron core with its shaft is utilized. The output data showing the final displacement is the effect on this shaft. The movement of rod is chosen in longitudinal manner that the negative impacts of eddy current losses are handled.

Working principle of this device have a reliance upon the rate of coupling between windings according to the position of core inside the bobbin. As can be seen from the construction, primary windings are fed with input signal and couple the secondary windings. The main calculations are based on the case whenever the location of core is the center, and coupling are halved among both windings (Formula 1). In the edge cases, winding, which is near to the core, its coupling rate is higher than the other one (Formula 2 and 3). In below noted formulas, SW characterizes the level of induced EMF in specific windings.

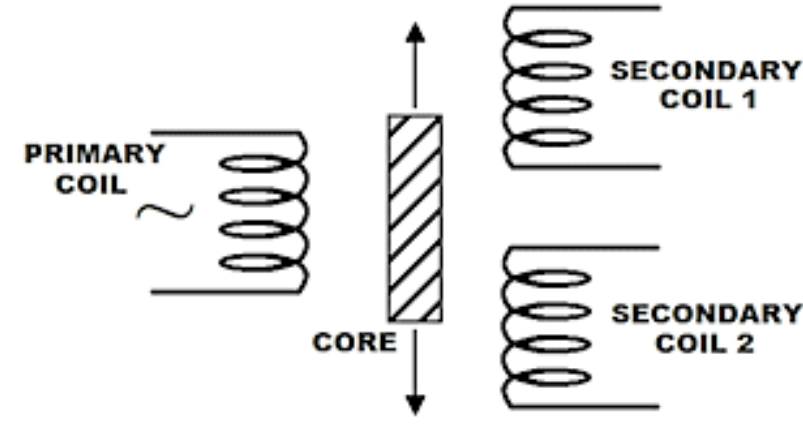

Figure 1. LVDT construction

$$
\begin{gathered}
S W 1=S W 2 \text { (1) } \\
S W 1<S W 2=>V_{e}<0 \text { (SW1 is near, movement is upward) } \\
S W 1>S W 2=>V_{e}>0 \text { (SW2 is near, movement is downward) }
\end{gathered}
$$

In the linear variable displacement sensors, the signal conditioning circuit characterizes the rate of both linearity and precision. This circuit consist of the signal generator, the demodulator, and the measurement device. As a signal generator, oscillators, producing sine and square wave signals, are mostly utilized. Manipulation of response time is handled by changing the oscillation frequency. Turning to the demodulators, they are used to convert input signal, which is alternating current, to direct current to observe by using measurement devices. This conversion is reached via parallelly connected resistors and capacitors right after the rectifier diodes. However, there are some types of LVDT whose input signal is also DC to handle above mentioned difficulties.

Speaking of demodulator [5], the traditional synchronous one requires a low-pass filter to remove the excitation signal. In the past, the envelope detector comprised diode is used to achieve the displacement signal. However, the threshold voltage of the diode causes the large error signal in the demodulated signal at the small amplitude of the LVDT signal. In addition, the envelope detector also requires the low-pass filter in the signal path. Unfortunately, the use of low-pass filter in the signal path of both the synchronous demodulator and the envelope detector is deteriorated the response time of the demodulator due to the large delay time caused by the dominant pole. Moreover, the frequency of the excitation signal is assigned as specific frequency to match the lowpass filter. The deviation from the specific frequency causes the error in the core-position signal. The use of the sample-and-hold circuit (SHC) can be prevented the error mentioned above. This is due to the behavior of the SHC that is in the form of 'sinc' function. The 'sinc' function exhibits the characteristic like a low-pass filter. The advantage of the use of the SHC to extract the core-position signal from the LVDT signal is that the low-pass filter can be avoided. Many applications using the SHC instead of the synchronous demodulator and the envelope detector. However, the control signal of the SHC is provided from the excitation signal that causes the error due to the phase shift between the excitation signal and the LVDT signal. To prevent this error, the peakamplitude finder is introduced to generate the control signal from the LVDT signal. The control signal is laid on the peak amplitude position of the LVDT signal and assigned as the narrow pulse width to minimize the ripple on the displacement signal. This technique avoids the phase shift mentioned above and can be operated with wide frequency 
range of the excitation signal. In this article, another circuit technique to extract the core-position signal from the LVDT signal using the integrator is proposed. The proposed circuit requires the integrator to average the half cycle of the LVDT signal. The resulting signal is linearly proportional to the core displacement. The proposed circuit is constructed without the specific devices. Only the commercially available devices are required. Therefore, the economical attraction is obtained.

In more general sayings, the application for this type of sensors are military, manufacturing, power turbines, and aircraft industry. For instance, the movement of actuators in the plane wings is normally analyzed via LVDTs. Another example is the measurement of off-axis movement of wheels by utilizing spring-loaded sensors. In the real-world applications, the main parameter of component, defining the level in which degree it is preferred, is dependent upon ratio between advantages and disadvantages. The main drawback for its use is the compulsion of shielding. However, the high level of linearity and resolution is counted as the primary benefits of application. While considering the low power consumption, less amount of friction (inexistence of contacting points), and the durability to the harsh conditions, it can easily be stated that these sensors would be an ultimate choice for the critical applications. Above noted term of harsh conditions generalizes the environment disturbances like vibrations and immediate temperature changes.

Like most electronic components, LVDTs have also some analogues and complementizes. For instance, angular (rotational) displacement is measured by using rotary variable differential transformer, called as RVDT, which is the counterpart to LVDTs. On the other hand, these sensors are more analogous to the "wall-wart" voltage transformer according to their working principles.

A simple read-out circuit for LVDT signal conditioning has been proposed. This method does not need complex building blocks like low-distortion sine wave oscillators for primary excitation and compensation circuitry for primary to secondary phase shifts. No separate excitation circuitry is required for secondary inductance measurement. With a rudimentary discrete component based Colpitts oscillator circuit and an FPGA circuit used merely for frequency counting, it has been shown that the resolution and linearity performance obtained is comparable to those obtained using sophisticated signal processing algorithms and/or signal conditioning ASICs. The performance can be optimized further by suitably selecting the oscillation frequency ranges, the supply voltages, increasing the frequency counter clock frequencies, and locking high frequency PLLs to the Colpitts oscillator.

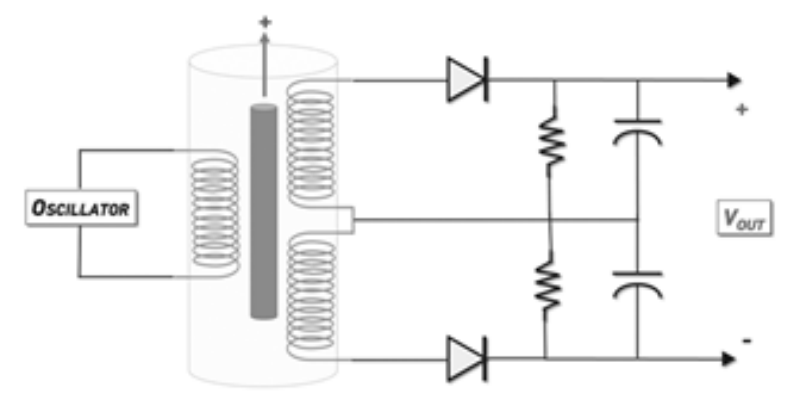

Figure 2. Signal conditioning circuit

\section{MAGNETOSTRICTIVE SENSORS}

Magnetostrictive sensor detects the linear position by using the permanent magnet. The primary element of this sensor is the sensing element, known as the waveguide. It is normally made of ferromagnetic material, for example the iron family (iron, cobalt, and nickel) and their alloys. In addition, position magnet, moving ring-shaped around the position magnet. The other operating part is the pulse detection system sensing the pulse and sending to the electronic circuit. Damping module is the component handling any environment effects, such as oscillations.

Working principle of this sensor depends upon the phenomenon which is called magnetostriction effect. This effect simply analyses the case where either shape or size of magnetic material is affected by the magnetic field. This change lasts up to magnetic saturation. This phenomenon was firstly tested by James Joule in 1842. It is also called Joule's effect. This process is characterized and analyzed by the magnetostriction coefficient which is equal to the ratio between the extension and the original length. Turning to the magnetostrictive sensors, rigid wire inside the probe tube serves to emit pulses as a current signal from electronic circuit of sensor in order to generate a circular magnetic field. The field created by the level transmitter magnetizes this wire in an axial manner. Superimposed two magnetic fields generate the bidirectional torsion wave along the wire. The crucial calculation relating to the measurement of the displacement is based upon the time difference between emission of current pulse and receive of the torsion wave in the probe head.

Like LVDTs, magnetostrictive sensors also offers noncontacting measurement that it solves the problem of existence of friction and requirement for the wear. Apart from that, high rate of linearity and the durability to the vibrations, created by the other operating devices, make this sensor more preferable. Even though these sensors can serve in infinitely high number of operating cycles, the main drawback is the artificial reduction of dead band by the manufacturers.

In terms of the direction of energy conversions, the applications of this sensor are adequately different. For instance, they are utilized for the production of force from the magnetic energy in the actuators. In the beginning of its uses, because of the lack of raw magnetostrictive materials its application included the simple things, such as sonar scanning devices, torque meters, and telephone receivers. With the use of the alloys, more challenging applications was come across. This can be positioners for adaptive optics, active vibration/noise control systems in both industrial and medical technology. The use of ultrasonic type in magnetostrictive transducers created new age in especially surgical paraphernalia and underwater sonar technology. 


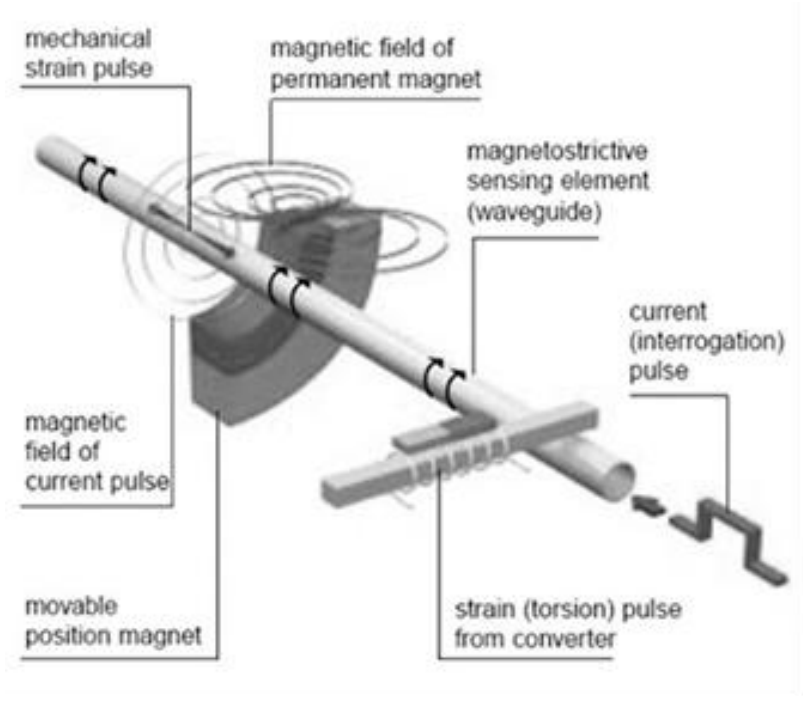

Figure 3. Magnetostrictive sensor construction

The magnetostrictive force sensor is a newly arisen sensor [15]. It utilizes the inverse magnetostrictive effect which refers to magnetization and permeability in ferromagnetic materials change caused by external force. It converts the force input into electromagnetic output. Magnetostrictive sensor has many promising features. Compared to the most common device for measuring force which is the bonded resistance strain gauge, it is relatively very rugged, and it is a durable force sensor for long term structural integrity. Compared to piezoelectric force sensor, it does not need an extra amplifying circuit or conversion circuit to process the output signal. Furthermore, it can measure the static and dynamic force simultaneously. It is these advantages that it has a broad range of applications including active suspensions and engine mounts for ground vehicles, active control of robots in manufacturing, active vibration control and monitoring, active control of buildings against seismic events, and monitoring overloads on bridges [3].

Nowadays, researchers have begun to do some researches on magnetostrictive sensors and obtained some achievements. Previous designs of magnetostrictive force sensors included work by Fleming and Kleinke and Uras [11]. Kleinke and Uras developed contacting and non-contacting magnetostrictive force sensors in which SAE 1018 steel was used as the sensitive material. A detection coil on the circuit produces a current due to the magnetic field. Strain on part or all of the ferromagnetic circuit changes the permeability, thus changing the amount of current in the detection coil. Unfortunately, this sensor is highly susceptible to electromagnetic interference, since stray magnetic field will also induce current in the detection coil. Baudendistel and Turner [6] studied a ring inverse magnetostrictive force sensor used a nickel-iron alloy as the core material. The reversing of the coil direction gives this sensor good insensitivity to electromagnetic interference. Dalian university of technology investigated a magnetostrictive force sensor used permalloy [7]. Unfortunately, magnetostrictive coefficient of these materials are smaller and the inverse magnetostrictive effect is poor [13].

\section{LINEAR ENCODER}

Linear encoder is a sensor encoding the position data. It reads the data as a scale and convert to the either digital or analog signal type. Certainly, this conversion can be carried out in the other direction, namely decoded data can be interpreted to the previous form. Linear encoders are classified into two subdivisions according to their assignments: incremental and absolute. Normally motion is analyzed by using the change in the position over the time. Optical, magnetic, inductive, capacitive, and eddy current are the technologies utilized in the linear encoders.

Applications for the linear encoders are divided into two primary subclasses. Firstly, coordinate-measuring machines, laser scanners, calipers, gear measurement, tension testers, and digital read outs(DROs). In terms of the high level of accuracy and speed, they are also preferred in motion systems, including robotics, machine tools, and printers.

Rapid advances in sensing and wireless technologies have led to a significant interest in understanding and development of wireless sensor networks (WSNs). Though easier to realize, wireless communication systems come at the price of lower channel capacity which results in higher quantization noise, packet loss and delay. Therefore, such communication constraints need to be considered in the design of WSNs. In the last decade or so, remote sensing and estimation in WSNs have witnessed significant progress in dynamical systems which evolve over time. In this work, we are interested in exploring the problem of remotely estimating the state of a stable stochastic scalar dynamic linear time-invariant (LTI) system (equivalently, a stationary Gauss-Markov process) that is being observed in the presence of noise by two sensors. From each terminal, the noisy measurement is encoded (preprocessed), quantized and then transmitted over a communication channel subject to packet erasures, to a remote node (or FC) for decoding and estimation purposes. In such system, we aim to design strategies for encoding the measurements of the sensors and decoding the state of the LTI system that allow improved estimation performance in the presence of packet erasures, quantization errors, process and measurement noises.

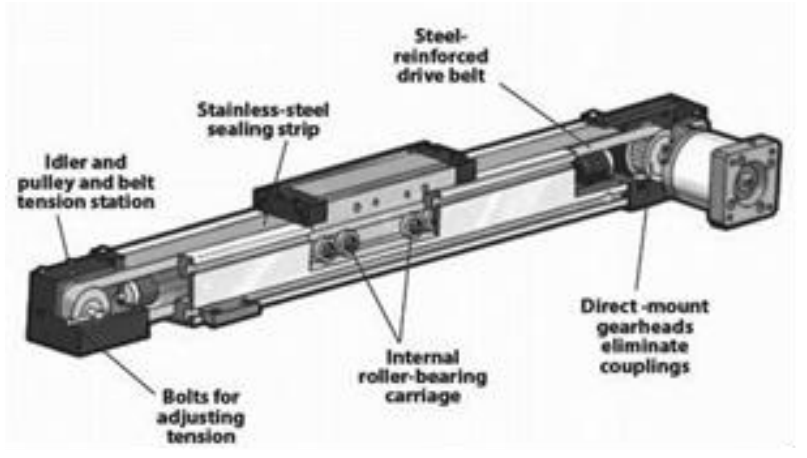

Figure 4. Linear encoder construction

Assuming Gaussian communication channels, linear encoding/decoding strategies are shown to be optimal with respect to minimizing a quadratic cost function in a singlesensor LTI system with Gaussian noise. The optimality, however, cannot be generalized to multi-sensor LTI systems. Linear schemes, although not optimal in multi-sensor setups, are easier to analyze. Moreover, finding the optimal non-linear policy is difficult in general. Hence, in this work, we focus on exploring linear encoding/decoding strategies that can be implemented with reasonable complexity. Under the model of channel erasures in a multi-sensor system (but without quantization noise), the authors in [7] obtain necessary and sufficient conditions for stabilization of an LTI system in mean-square sense by identifying an encoding algorithm, where each sensor has access to channel erasure information of all sensors. In [8], the authors proposed a linear 
encoding/decoding scheme for a single-sensor system setup when channel erasures are considered along with quantization noise. An important result of [8] is that the optimal strategy among all possible linear encoders corresponds to the transmission of the Kalman filter innovations under the assumption that the encoder has perfect knowledge of the channel. In this work, we generalize the result of [8] by considering two sensors and correspondingly two transmission links to the receiver. The results out of this generalization, as will be shown later, are non-trivial. Note that although we consider only two sensors, the results derived here can be easily generalized to any number of sensors. In particular, we propose two benchmark methods where the first is based on the fact that a perfect feedback link is available from the decoder to the encoders, and in the second benchmark method, besides the perfect receiver feedback, we assume that sensors are collocated such that encoders can be designed in a centralized manner [14]. Due to resource constraints, channel feedback may not be always available, and sensors are generally not co-located in distributed remote estimation applications. Therefore, we also propose distributed encoding/decoding schemes, where the encoders are not aware of the receiver state estimate. We propose a soft-innovation forwarding (SIF) scheme where each encoder transmits a convex combination of transmitting the state estimate at the encoder (state-forwarding (SF)) and the difference between encoder state estimate and the predicted state estimate at the receiver (innovation forwarding (IF)). Although the SIF strategy has a higher design complexity compared to SF or IF, its performance is superior to the latter methods for low erasure probabilities. The numerical simulations of authors in [8] also show that the encoding-decoding designs with receiver feedback considerably improve the performance compared to the case where the feedback is not available. The rest of the paper is organized as follows.

\section{THEORETICAL CONSIDERATION}

To achieve high precision in the operation of the Micrus sensor, several aspects of the generation, propagation, and reception of the ultrasonic signals, as well as their processing for the optimal estimation of the time delay, have to be considered. We put special emphasis on the aspects that distinguish Micrus from already existing MS sensors.

\section{Principle of Position Estimation}

The operation of the Micrus sensor is shown in Fig. 1. It consists of a long thin ferromagnetic tube, along which the cursor can move without contact. When an intense current pulse at ultrasonic frequencies $\mathrm{v} 0(\mathrm{t})$ is put through a cursor coil that is concentric with the tube, the MS effect causes a mechanical deformation in the tube at position $\mathrm{z}$ of the cursor. This deformation splits into two ultrasonic waves, which propagate at the speed of sound in the metal toward the ends of the waveguide, where they are received by piezoelectric transducers, producing signals $\mathrm{v} 1(\mathrm{t})$ and $\mathrm{v} 2(\mathrm{t})$. The cursor position can then be computed from the propagation time of the original ultrasonic signal to either of the received signals at the ends of the tube. For example, if we use time delay D12 between signals $\mathrm{v} 1(\mathrm{t})$ and $\mathrm{v} 2(\mathrm{t})$, position $\mathrm{z}$ is found to be

$$
\hat{z}=\frac{1}{2}\left(L-c \widehat{D}_{12}\right)
$$

where $\mathrm{L}$ is the total length of the tube, $\mathrm{c}$ is the speed of sound, and $\mathrm{z}^{\wedge}$ is the estimation of linear position $\mathrm{z}$.

\section{Behavior of the Transmitting Element}

A waveguide of cylindrical symmetry, such as a rod or a tube, is able to simultaneously support a number of propagating modes [8], [9], which, according to their spatial symmetry characteristics, are classified into torsional [denoted as $\mathrm{T}(0, \mathrm{~m})]$, longitudinal $[\mathrm{L}(0, \mathrm{~m})]$, and flexural $[\mathrm{F}(\mathrm{n}, \mathrm{m})]$. Usually, devices designed for the applications of ultrasonic waves in solids seek to excite a single propagating mode [10].

While commercial MS sensor designs exploit the first torsional mode $\mathrm{T}(0,1)$ of the waveguide, in this paper, we will focus on the first longitudinal mode $\mathrm{L}(0,1)$ for position measurement. A sensor design that excites this mode permits high transduction efficiency in both generation and reception, which, as will be shown in Section II-D, is important for accurate position estimation.

In general, the propagation speed of each mode depends on the frequency, which is a phenomenon called dispersion

$$
c_{\mathrm{ph}}(f)=\frac{2 \pi f}{k(f)}
$$

The computed dispersion curves for the low-frequency propagating modes in the tube used in the Micrus sensor are shown in Fig. 2(a), from which it can be seen that, unlike the case of the first torsional mode $\mathrm{T}(0,1)$, the longitudinal mode $\mathrm{L}(0,1)$ is dispersive.

If not all the frequency components of the signal travel at the same phase speed, the shape of the signal will change as it propagates along the waveguide. Because these signals are used for the estimation of position, the result is a systematic (or position-dependent) error in the measurement.

Several precautions need to be taken to minimize the dispersive effects. The phase speed of the $\mathrm{L}(0,1)$ mode is relatively constant from zero frequency up to the cutoff frequency of the second longitudinal mode $\mathrm{L}(0,2)$ (Fig. 2). Since this cutoff frequency inversely depends on the thickness of the tube, the thinnest possible tube should be used as the transmitting element. Likewise, most of the power spectrum of the excitation signal $v 0(t)$ should be contained in that nondispersive area. A waveform that is well suited for ultrasonic excitation is a sine train modulated by a Hanning window [12]

$$
v 0(t)=v e(t) \sin 2 \pi f 0 t
$$

where the envelope is

$$
v_{e}(t)=\frac{1}{2}[1-\cos (2 \pi t / T)]\left[S_{H}(t)-S_{H}(t-T)\right]
$$

$\mathrm{SH}(\mathrm{t})$ is Heaviside's step function, ncyc is the number of cycles of the signal, and $\mathrm{T}=\mathrm{ncyc} / \mathrm{fO}$ is its total length. In Fig. 2(b), we plot the power spectrum P0(f) for the waveform of (3) (with ncyc $=6$ and $\mathrm{f0}=60 \mathrm{kHz}$, which are the parameters used for the Micrus sensor), which shows that the phase speed only varies by $9 \mathrm{~m} / \mathrm{s}$ over the $15-\mathrm{kHz}$ bandwidth of the signal. We will evaluate the influence of dispersion in the Micrus sensor in Section IV.

\section{CONCLUSION}

In this paper, we have examined the physical factors that limit the measurement precision that is obtainable with MS linear position sensors and have proposed an alternative design to achieve better performance. The results with a prototype MS sensor built according to those considerations have shown an accuracy improvement of about six times over those of 
commercial models. Our sensor specifications are summarized in Table II. We believe that the precision is ultimately limited by the mechanical and magnetic homogeneity of the tube, which serves as a waveguide for the propagation of the ultrasonic signals. The error pattern obtained suggests that further improvements in the position sensor are possible and that the precision of optical encoders may be reached with the MS technology when the publications are assembled.

\section{ACKNOWLEDGMENTS}

All authors would like to express their gratitude to the PostGraduate Program of Studies "Automation of Productions and services" of PUAS, for the financial support to undertake this research project.

\section{REFERENCES}

[1] B. Corona, M. Nakano, H. Pérez, "Adaptive Watermarking Algorithm for Binary Image Watermarks", Lecture Notes in Computer Science, Springer, pp. 207-215, 2004.

[2] A. A. Reddy and B. N. Chatterji, "A new wavelet based logo-watermarking scheme," Pattern Recognition Letters, vol. 26, pp. 1019-1027, 2005.

[3] P. S. Huang, C. S. Chiang, C. P. Chang, and T. M. Tu, "Robust spatial watermarking technique for colour images via direct saturation adjustment," Vision, Image and Signal Processing, IEE Proceedings -, vol. 152, pp. 561-574, 2005.

[4] F. Gonzalez and J. Hernandez, " A tutorial on Digital Watermarking ", In IEEE annual Carnahan conference on security technology, Spain, 1999.

[5] D. Kunder, "Multi-resolution Digital Watermarking Algorithms and Implications for Multimedia Signals", Ph.D. thesis, university of Toronto, Canada, 2001.
[6] J. Eggers, J. Su and B. Girod," Robustness of a Blind Image Watermarking Scheme", Proc. IEEE Int. Conf. on Image Proc., Vancouver, 2000.

[7] Barni M., Bartolini F., Piva A., Multichannel watermarking of color images, IEEE Transaction on Circuits and Systems of Video Technology 12(3) (2002) 142-156.

[8] Kundur D., Hatzinakos D., Towards robust logo watermarking using multiresolution image fusion, IEEE Transcations on Multimedia 6 (2004) 185-197.

[9] C.S. Lu, H.Y.M Liao, "Multipurpose watermarking for image authentication and protection," IEEE Transaction on Image Processing, vol. 10, pp. 1579-1592, Oct. 2001.

[10] L. Ghouti, A. Bouridane, M.K. Ibrahim, and S. Boussakta, "Digital image watermarking using balanced multiwavelets", IEEE Trans. Signal Process., 2006, Vol. 54, No. 4, pp. 1519-1536.

[11] P. Tay and J. Havlicek, "Image Watermarking Using Wavelets", in Proceedings of the 2002 IEEE, pp. II.258 II.261, 2002.

[12] P. Kumswat, Ki. Attakitmongcol and A. Striaew, "A New Approach for Optimization in Image Watermarking by Using Genetic Algorithms", IEEE Transactions on Signal Processing, Vol. 53, No. 12, pp. 4707-4719, December, 2005.

[13] H. Daren, L. Jifuen,H. Jiwu, and L. Hongmei, "A DWTBased Image Watermarking Algorithm", in Proceedings of the IEEE International Conference on Multimedia and Expo, pp. 429-432, 2001.

[14] C. Hsu and J. Wu, "Multi-resolution Watermarking for Digital Images", IEEE Transactions on Circuits and Systems- II, Vol. 45, No. 8, pp. 1097-1101, August 1998.

[15] R. Mehul, "Discrete Wavelet Transform Based Multiple Watermarking Scheme", in Proceedings of the 2003 IEEE TENCON, pp. 935-938, 2003. 